\title{
ON THE BIHARMONICITY OF PRODUCT MAPS
}

\author{
LEONARD TODJIHOUNDE
}

Received 29 November 2005; Revised 9 May 2006; Accepted 11 May 2006

We introduce the warped product of maps defined between Riemannian warped product spaces and we give necessary and sufficient conditions for warped product maps to be (bi)harmonic. We obtain then some characterizations of nontrivial harmonic metrics and nonharmonic biharmonic metrics on warped product spaces.

Copyright (c) 2006 Hindawi Publishing Corporation. All rights reserved.

\section{Introduction}

Let $f:\left(M^{m}, g\right) \rightarrow\left(N^{n}, h\right)$ be a map between the $m$-dimensional Riemannian manifold $(M, g)$ and the $n$-dimensional Riemannian manifold $(N, h)$.

The energy of the map $f$ is given by

$$
E(f)=\int_{M} e(f) v_{g}
$$

where $v_{g}$ is the volume form on $(M, g)$ and $e(f)(x):=(1 / 2)\|d f(x)\|_{T^{*} M \otimes f^{-1} T N}^{2}$ is the energy density of $f$ at the point $x \in M$. In local coordinates $\left(x^{i}\right)_{i=1}^{m}$ on $M$ and $\left(y^{a}\right)_{a=1}^{n}$ on $N$, the energy density is given by $e(f)(x)=(1 / 2) g^{i j}(x) h_{a b}(f(x))\left(\partial f^{a} / \partial x^{i}\right)\left(\partial f^{b} / \partial x^{j}\right)$.

Critical points of the energy functional are called harmonic maps.

The first variational formula of the energy gives the following characterization of harmonic maps: the map $f$ is harmonic if and only if its tension field $\tau(f)$ vanishes identically, where the tension field is given by

$$
\tau(f):=\operatorname{trace}_{g} \nabla d f=g^{i j}\left(\frac{\partial^{2} f^{a}}{\partial x^{i} \partial x^{j}}-{ }^{M} \Gamma_{i j}^{k} \frac{\partial f^{a}}{\partial x^{k}}+{ }^{N} \Gamma_{b c}^{a} \frac{\partial f^{b}}{\partial x^{i}} \frac{\partial f^{c}}{\partial x^{j}}\right) \frac{\partial}{\partial y^{a}}
$$

with ${ }^{M} \Gamma_{i j}^{k}$ and ${ }^{N} \Gamma_{b c}^{a}$ being the Christoffel symbols of the metrics $g$ and $h$, respectively. The theory of harmonic maps was introduced by Eells and Sampson [7] and has been 
developed by many authors. The bienergy $E_{2}(f)$ of the map $f$ is defined by

$$
E_{2}(f):=\frac{1}{2} \int_{M}\|\tau(f)\|^{2} v_{g}
$$

As it was observed in [1], the bienergy is the first variation of the energy with respect to a particular variation of the map; precisely that with $-\tau(f)$ as variational vector field.

The map $f$ is said to be biharmonic if it is a critical point of the bienergy functional. The Euler-Lagrange equation associated to the bienergy functional is given by

$$
\tau_{2}(f):=-\Delta^{f} \tau(f)-\operatorname{trace}_{g} R^{N}(d f, \tau(f)) d f=0,
$$

where $\Delta^{f}=-\operatorname{trace}_{g}\left(\nabla^{f} \nabla^{f}-\nabla_{\nabla}^{f}\right)$ is the Laplacian on the sections of $f^{-1}(T N)$, and $R^{N}$ is the Riemannian curvature operator of $(N, h)$ (see $[8,9])$.

Note that $\tau_{2}(f)=-J_{f}(\tau(f))$, where $J_{f}$ is the Jacobi operator of $f$, which gives the second variation of the energy functional at its critical (harmonic) points.

Harmonic maps are obviously biharmonic and are absolute minimum of the bienergy. In [9], the author proved that harmonicity and biharmonicity are equivalent if $M$ is compact and $R^{N} \leq 0$, or if $f$ is a Riemannian immersion with $\|\tau(f)\|$ constant (see [11]) and $R^{N} \leq 0$.

Examples of nonharmonic biharmonic maps are given in [5] and also in [8], where it is proved that the generalized Clifford torus $\mathbb{P} p(1 / \sqrt{2}) \times \mathbb{S} q(1 / \sqrt{2}) \rightarrow \mathbb{S}^{m+1}$ with $p+q=m$, $p \neq q$, is a nonharmonic biharmonic submanifold of $\mathbb{S}^{m+1}$.

A classification of nonharmonic biharmonic submanifolds of $\mathbb{S}^{3}$ is given in $[3,4]$.

The method of conformal deformation of metrics has been used by several authors to study the existence or properties of (bi)harmonic maps.

In the case of warped or twisted product spaces, the same techniques can be used, not by deforming conformally the whole metric but by acting only on the warping or twisting functions. In the present paper we consider a particular class of maps defined between warped product spaces, the so-called warped product maps, and we examine (bi)harmonic properties of these maps in relation to that of the component maps. We apply the results obtained to characterize (bi)harmonic metrics on warped product spaces.

Throughout the paper, manifolds, metrics, and maps are assumed to be smooth.

\section{Harmonicity of warped product maps, harmonic metrics, and nonharmonic biharmonic metrics on warped product spaces}

2.1. Warped product of manifolds. Let $(M, g)$ and $(N, h)$ be two Riemannian manifolds of dimensions $m$ and $n$, respectively, and $\lambda \in C^{\infty}(M)$ a strictly positive function on $M$.

The warped product of $(M, g)$ and $(N, h)$, with warping function $\lambda$, is the product manifold $M \times N$ endowed with the metric $G_{\lambda}$ defined by

$$
G_{\lambda}=: \pi^{*} g+(\lambda \circ \pi)^{2} \sigma^{*} h
$$

where $\pi$ and $\sigma$ are the projections of $M \times N$ on $M$ and $N$, respectively.

The metric $G_{\lambda}$ is also called the warped product of the metrics $g$ and $h$ with warping function $\lambda$. 
For $X, Y \in \mathscr{b}(T M)$ and $U, V \in \mathscr{C}(T N)$,

$$
G_{\lambda}(X+U, Y+V)=g(X, Y)+(\lambda \circ \pi)^{2} h(U, V),
$$

where we use the same convention as in [10] for the notations of vector fields.

Let ${ }^{g} \nabla,{ }^{h} \nabla$, and $\nabla$ be the Levi-Civita connections of $(M, g),(N, h)$, and $\left(M \times N, G_{\lambda}\right)$, respectively. We have the following (see [10]).

Lemma 2.1. For $X, Y \in \mathscr{C}(T M)$ and $U, V \in \mathscr{C}(T N)$, it holds that

(i) $\pi_{*}\left(\nabla_{X} Y\right)=g \nabla_{X} Y$ and $\sigma_{*}\left(\nabla_{X} Y\right)=0$,

(ii) $\nabla_{X} U=\nabla_{U} X=(d \lambda(X) / \lambda) U$,

(iii) $\pi_{*}\left(\nabla_{U} V\right)=-(\langle U, V\rangle / \lambda) \operatorname{grad} \lambda=-\lambda h(U, V) \operatorname{grad} \lambda$,

(iv) $\sigma_{*}\left(\nabla_{U} V\right)={ }^{h} \nabla_{U} V$.

Let $\left(X_{1}, X_{2}, \ldots, X_{m+n}\right)=\left(x_{1}, x_{2}, \ldots, x_{m}, u_{1}, u_{2}, \ldots, u_{n}\right)$ be a local coordinates system in $M \times N$, where $\left(x_{1}, x_{2}, \ldots, x_{m}\right)$ and $\left(u_{1}, u_{2}, \ldots, u_{n}\right)$ are local coordinates systems in $M$ and $N$, respectively. We use the indices notations $\hat{I}, \hat{J}, \hat{K}, \ldots \in\{1, \ldots, m+n\}$ on $M \times N, i, j, k, \ldots \in$ $\{1, \ldots, m\}$ on $M$, and $\bar{i}, \bar{j}, \bar{k}, \ldots \in\{1, \ldots, n\}$ on $N$ and for $\hat{I}, \hat{J}, \hat{K}, \ldots \in\{1, \ldots, m\}$, we put $\hat{I}=i, \hat{J}=j, \hat{K}=k, \ldots$; for $\hat{I}, \hat{J}, \hat{K}, \ldots \in\{m, \ldots, m+n\}$, we put $\hat{I}=m+\bar{i} \equiv \bar{i}, \hat{J}=m+\bar{j} \equiv \bar{j}$, $\hat{K}=m+\bar{k} \equiv \bar{k}$. We have

$$
G_{\lambda}=\left(G_{\lambda \hat{I J}}\right)=\left(\begin{array}{cc}
g_{i j} & 0 \\
0 & \lambda^{2} h_{i \bar{j}}
\end{array}\right), \quad G_{\lambda}^{-1}=\left(G_{\lambda}^{\widehat{I} \hat{J}}\right)=\left(\begin{array}{cc}
g^{i j} & 0 \\
0 & \lambda^{-2} h^{\bar{i} j}
\end{array}\right) .
$$

Let ${ }^{1} \Gamma_{i j}^{k},{ }^{2} \Gamma_{\bar{i} \dot{j}}^{\bar{k}}$, and $\Gamma_{\widehat{I} \hat{J}}^{\widehat{K}}$ be Christoffel's symbols on $(M, g),(N, h)$, and $\left(M \times N, G_{\lambda}\right)$, respectively.

A direct computation from Lemma 2.1 leads to the following (see, e.g., [12, page 111]).

Lemma 2.2. (i) $\Gamma_{i j}^{k}={ }^{1} \Gamma_{i j}^{k}$, for all $i, j, k \in\{1, \ldots, m\}$.

(ii) $\Gamma_{i \bar{j}}^{k}=\Gamma_{i j}^{\bar{k}}=0$, for all $i, j, k \in\{1, \ldots, m\}$ and for all $\bar{j}, \bar{k} \in\{1, \ldots, n\}$.

(iii) $\Gamma_{i \bar{j}}^{\bar{k}}=\left(\partial \log \lambda / \partial x_{i}\right) \delta_{\bar{j}}^{\bar{k}}$, for all $i \in\{1, \ldots, m\}$ and for all $\bar{j}, \bar{k} \in\{1, \ldots, n\}$.

(iv) $\Gamma_{\bar{i} \bar{j}}^{k}=-(1 / 2)\left(\operatorname{grad} \lambda^{2}\right)^{k} h_{\bar{i} \bar{j}}$, for all $k \in\{1, \ldots, m\}$ and for all $\bar{i}, \bar{j} \in\{1, \ldots, n\}$.

(v) $\Gamma_{\bar{i} \bar{j}}^{\bar{k}}={ }^{2} \Gamma_{\bar{i} \bar{j}}^{\bar{k}}$, for all $\bar{i}, \bar{j}, \bar{k} \in\{1, \ldots, n\}$.

The relations (i)-(v) of Lemma 2.2 can be also obtained directly from the formula

$$
\Gamma_{\widehat{I} \hat{J}}^{\hat{K}}=\frac{1}{2} G_{\lambda}^{\hat{K} \hat{L}}\left(\frac{\partial G_{\lambda \widehat{I} \hat{L}}}{\partial X_{\hat{J}}}+\frac{\partial G_{\lambda \widehat{J}}}{\partial X_{\widehat{I}}}-\frac{\partial G_{\lambda \widehat{I}}}{\partial X_{\widehat{L}}}\right)
$$

2.2. Warped product maps. Let $\left(M, G_{\lambda}\right)$ be the warped product of two Riemannian manifolds $\left(M_{1}, g_{1}\right)$ and $\left(M_{2}, g_{2}\right)$ with warping function $\lambda \in C^{\infty}\left(M_{1}\right)$, and let $\left(N, H_{\varrho}\right)$ be the warped product of the Riemannian manifolds $\left(N_{1}, h_{1}\right)$ and $\left(N_{2}, h_{2}\right)$ with warping function $\varrho \in C^{\infty}\left(N_{1}\right)$.

Let $\phi_{1}:\left(M_{1}, g_{1}\right) \rightarrow\left(N_{1}, h_{1}\right)$ and $\phi_{2}:\left(M_{2}, g_{2}\right) \rightarrow\left(N_{2}, h_{2}\right)$ be two maps. 
4 On the biharmonicity of product maps

Defintion 2.3. The warped product of $\phi_{1}$ and $\phi_{2}$, with warping functions $\lambda$ and $\varrho$, is defined as the map $\Phi_{\lambda, \varrho}$ by

$$
\begin{gathered}
\Phi_{\lambda, \varrho}:\left(M, G_{\lambda}\right) \longrightarrow\left(N, H_{\varrho}\right), \\
\left(P_{1}, P_{2}\right) \longmapsto\left(\phi_{1}\left(P_{1}\right), \phi_{2}\left(P_{2}\right)\right) .
\end{gathered}
$$

The maps $\phi_{1}$ and $\phi_{2}$ are then called the components of $\Phi_{\lambda, \varrho}$.

Let $\left(X_{1}, X_{2}, \ldots, X_{m_{1}+m_{2}}\right)=\left(x_{1}, x_{2}, \ldots, x_{m_{1}}, u_{1}, u_{2}, \ldots, u_{m_{2}}\right)$ and $\left(Y_{1}, Y_{2}, \ldots, Y_{n_{1}+n_{2}}\right)=\left(y_{1}\right.$, $\left.y_{2}, \ldots, y_{n_{1}}, v_{1}, v_{2}, \ldots, v_{n_{2}}\right)$ be local coordinates systems in $M_{1} \times M_{2}$ and $N_{1} \times N_{2}$, respectively, defined as above with the same notations' conventions for the indices for which we use $\hat{I}, \hat{J}, \hat{K}$ on $M_{1} \times M_{2} ; i, j, k$ on $M_{1} ; \bar{i}, \bar{j}, \bar{k}$ on $M_{2} ; \hat{A}, \hat{B}, \hat{C}$ on $N_{1} \times N_{2} ; a, b, c$ on $N_{1}$, and $\bar{a}, \bar{b}, \bar{c}$ on $N_{2}$.

The second fundamental form of the warped product map $\Phi_{\lambda, \varrho}$ of $\phi_{1}$ and $\phi_{2}$ is given by the following.

Lemma 2.4. (i) For $i, j \in\left\{1, \ldots, m_{1}\right\}$ and $a \in\left\{1, \ldots, n_{1}\right\}$,

$$
\left(\nabla d \Phi_{\lambda, \varrho}\right)_{i j}^{a}=\left({ }^{1} \nabla d \phi_{1}\right)_{i j}^{a}
$$

(ii) For $i, j \in\left\{1, \ldots, m_{1}\right\}, a \in\left\{1, \ldots, n_{1}\right\}, \bar{j} \in\left\{1, \ldots, m_{2}\right\}$, and $\bar{a} \in\left\{1, \ldots, n_{2}\right\}$,

$$
\left(\nabla d \Phi_{\lambda, \varrho}\right)_{i j}^{\bar{a}}=\left(\nabla d \Phi_{\lambda, \varrho}\right)_{i \bar{j}}^{a}=0 .
$$

(iii) For $i \in\left\{1, \ldots, m_{1}\right\}, \bar{j} \in\left\{1, \ldots, m_{2}\right\}$, and $\bar{a} \in\left\{1, \ldots, n_{2}\right\}$,

$$
\left(\nabla d \Phi_{\lambda, \varrho}\right)_{i \bar{j}}^{\bar{a}}=-\frac{\partial \log \lambda}{\partial x_{i}} \cdot \frac{\partial \phi_{2}^{\bar{a}}}{\partial u_{j}}+\frac{\partial \log \varrho}{\partial y_{b}} \cdot \frac{\partial \phi_{1}^{b}}{\partial x_{i}} \cdot \frac{\partial \phi_{2}^{\bar{a}}}{\partial u_{j}} .
$$

(iv) For $\bar{i}, \bar{j} \in\left\{1, \ldots, m_{2}\right\}$ and $a \in\left\{1, \ldots, n_{1}\right\}$,

$$
\left(\nabla d \Phi_{\lambda, \varrho}\right)_{\bar{i} \bar{j}}^{a}=\frac{1}{2}\left(\operatorname{grad} \lambda^{2}\right)^{k} \frac{\partial \phi_{1}^{a}}{\partial x_{k}} g_{2 i \bar{j}}-\frac{1}{2}\left(\operatorname{grad} \varrho^{2}\right)^{a} \frac{\partial \phi_{2}^{\bar{b}}}{\partial u_{\bar{i}}} \cdot \frac{\partial \phi_{2}^{\bar{c}}}{\partial u_{\bar{j}}} h_{2 \bar{b} \bar{c}} .
$$

(v) For $\bar{i}, \bar{j} \in\left\{1, \ldots, m_{2}\right\}$ and $\bar{a} \in\left\{1, \ldots, n_{2}\right\}$,

$$
\left(\nabla d \Phi_{\lambda, \varrho}\right)_{\bar{i} \bar{j}}^{\bar{a}}=\left({ }^{2} \nabla d \phi_{2}\right)_{\bar{i} \bar{j}}^{\bar{a}} .
$$

Let $\tau\left(\Phi_{\lambda, \varrho}\right), \tau\left(\phi_{1}\right)$, and $\tau\left(\phi_{2}\right)$ be the tension fields of the warped product map $\Phi_{\lambda, \varrho}$ and of its components $\phi_{1}$ and $\phi_{2}$. From Lemma 2.4 we have the following proposition.

Proposition 2.5.

$$
\tau\left(\Phi_{\lambda, \varrho}\right)=\tau\left(\phi_{1}\right)+\lambda^{-2} \tau\left(\phi_{2}\right)+\lambda^{-2}\left[\frac{m_{2}}{2} d \phi_{1}\left(\operatorname{grad}_{M_{1}} \lambda^{2}\right)-\mathrm{e}\left(\phi_{2}\right)\left(\operatorname{grad}_{N_{1}} \varrho^{2}\right) \circ \phi_{1}\right],
$$

with $d \phi_{1}\left(\operatorname{grad}_{M_{1}} \lambda^{2}\right)=\left[d \phi_{1}^{a}\left(\operatorname{grad}_{M_{1}} \lambda^{2}\right)\right]\left(\partial / \partial y^{a}\right)$, where $\mathrm{e}\left(\phi_{2}\right)$ is the energy density of $\phi_{2}$, and $\operatorname{grad}_{M_{1}}$ and $\operatorname{grad}_{N_{1}}$ denote the gradient operators on $M_{1}$ and $N_{1}$, respectively. 
Proof. We have

$$
\begin{aligned}
\tau\left(\Phi_{\lambda, \varrho}\right)= & G_{\lambda}^{\hat{I} \hat{J}}\left(\nabla d \Phi_{\lambda, \varrho}\right)_{\hat{I} \hat{\jmath}}^{\hat{A}} \frac{\partial}{\partial Y_{\hat{A}}}=G_{\lambda}^{i j}\left(\nabla d \Phi_{\lambda, \varrho}\right)_{i j}^{a} \frac{\partial}{\partial y_{a}}+G_{\lambda}^{i j}\left(\nabla d \Phi_{\lambda, \varrho}\right)_{i j}^{\bar{a}} \frac{\partial}{\partial v_{\bar{a}}} \\
& +G_{\lambda}^{\bar{i} \bar{j}}\left(\nabla d \Phi_{\lambda, \varrho}\right)_{\bar{i} \bar{j}}^{a} \frac{\partial}{\partial y_{a}}+G_{\lambda}^{\bar{i} \bar{j}}\left(\nabla d \Phi_{\lambda, \varrho}\right)_{\bar{i} \bar{j} \frac{\partial}{\partial v_{\bar{a}}}}^{\bar{\partial}} \\
= & g_{1}^{i j}\left({ }^{1} \nabla d \phi_{1}\right)_{i j}^{a} \frac{\partial}{\partial y_{a}}+\frac{1}{2} \lambda^{-2} g_{2}^{\bar{i} \bar{j}}\left(\operatorname{grad}_{M_{1}} \lambda^{2}\right)^{k} \frac{\partial \phi_{1}^{a}}{\partial x_{k}} g_{2 \bar{i} \bar{j}} \frac{\partial}{\partial y_{a}} \\
& -\frac{1}{2} \lambda^{-2} g_{2}^{\bar{i} \bar{j}}\left(\operatorname{grad}_{N_{1}} \varrho^{2}\right)^{a} \frac{\partial \phi_{2}^{\bar{b}}}{\partial u_{\bar{i}}} \cdot \frac{\partial \phi_{2}^{\bar{c}}}{\partial u_{\bar{j}}} h_{2 \bar{b} \bar{c}} \frac{\partial}{\partial y_{a}}+\lambda^{-2} g_{2}^{\bar{i} \bar{j}}\left({ }^{2} \nabla d \phi_{2}\right)_{\bar{i} \bar{j}}^{\bar{a}} \frac{\partial}{\partial v_{\bar{a}}} .
\end{aligned}
$$

By evaluating each term of the second hand member of the last equality, we obtain

$$
\begin{gathered}
g_{1}^{i j}\left({ }^{1} \nabla d \phi_{1}\right)_{i j}^{a} \frac{\partial}{\partial y_{a}}=\tau\left(\phi_{1}\right), \\
\frac{1}{2} \lambda^{-2} g_{2}^{i \bar{j}}\left(\operatorname{grad}_{M_{1}} \lambda^{2}\right)^{k} \frac{\partial \phi_{1}^{a}}{\partial x_{k}} g_{2 i \bar{j} \frac{\partial}{\partial y_{a}}}=\lambda^{-2} \frac{m_{2}}{2}\left[d \phi_{1}\left(\operatorname{grad}_{M_{1}} \lambda^{2}\right)\right], \\
\frac{1}{2} \lambda^{-2} g_{2}^{i \bar{j}}\left(\operatorname{grad}_{N_{1}} \varrho^{2}\right)^{a} \frac{\partial \phi_{2}^{\bar{b}}}{\partial u_{\bar{i}}} \cdot \frac{\partial \phi_{2}^{\bar{c}}}{\partial u_{\bar{j}}} h_{2 \bar{b} \bar{c}} \frac{\partial}{\partial y_{a}}=\lambda^{-2} \mathrm{e}\left(\phi_{2}\right)\left[\left(\operatorname{grad}_{N_{1}} \varrho^{2}\right) \circ \phi_{1}\right], \\
\lambda^{-2} g_{2}^{\bar{i} \bar{j}}\left({ }^{2} \nabla d \phi_{2}\right)_{\bar{i} \bar{j} \frac{\partial}{\partial v_{\bar{a}}}}^{\bar{a}}=\lambda^{-2} \tau\left(\phi_{2}\right) .
\end{gathered}
$$

Thus we get the result.

The following result is a consequence of the previous proposition.

Corollary 2.6. Let $\phi_{1}:\left(M_{1}, g_{1}\right) \rightarrow\left(N_{1}, h_{1}\right)$ and $\phi_{2}:\left(M_{2}, g_{2}\right) \rightarrow\left(N_{2}, h_{2}\right)$ be two harmonic maps. The warped product $\Phi_{\lambda, \varrho}$ of $\phi_{1}$ and $\phi_{2}$, with warping functions $\lambda \in C^{\infty}\left(M_{1}\right)$ and $\varrho \in C^{\infty}\left(N_{1}\right)$, is harmonic if and only if

$$
\frac{m_{2}}{2} d \phi_{1}\left(\operatorname{grad}_{M_{1}} \lambda^{2}\right)=\mathrm{e}\left(\phi_{2}\right)\left(\operatorname{grad}_{N_{1}} \varrho^{2}\right) \circ \phi_{1}
$$

2.3. Harmonic metrics on warped product spaces. Let us first recall the definition of harmonic metrics.

Defintion 2.7. Let $(M, g)$ be a Riemannian manifold. A metric $G$ on $M$ is said to be harmonic with respect to $g$ if the identity id $:(M, g) \rightarrow(M, G)$ is a harmonic map (see [6] for more information about harmonic metrics).

Let $\left(M \times N, G_{\lambda}\right)$ be the warped product of two Riemannian manifolds $(M, g)$ and $(N, h)$ with warping function $\lambda \in C^{\infty}(M), \lambda>0$.

Let $G_{\varrho}$ be the metric defined on $M \times N$ by $G_{\varrho}=\pi^{*} g+(\varrho \circ \pi)^{2} \sigma^{*} h$, with $\varrho \in C^{\infty}(M)$, $\varrho>0$, where $\pi$ and $\sigma$ are as usual the projections of $M \times N$ on $M$ and $N$, respectively. The following holds. 
6 On the biharmonicity of product maps

Theorem 2.8. Assume $M$ is connected.

The metric $G_{\varrho}$ is harmonic with respect to $G_{\lambda}$ if and only if

$$
\lambda^{2}-\varrho^{2} \text { is a constant function on } M \text {. }
$$

Proof. Let $\Phi_{\lambda, \varrho}:\left(M \times N, G_{\lambda}\right) \rightarrow\left(M \times N, G_{\varrho}\right)$ be the warped product of the identity maps $\operatorname{id}_{M}:(M, g) \rightarrow(M, g)$ and id $N:(N, h) \rightarrow(N, h)$, with warping functions $\lambda$ and $\varrho$.

The metric $G_{\varrho}$ is harmonic with respect to $G_{\lambda}$ if and only if $\mathscr{I}_{\lambda, \varrho}$ is a harmonic map.

Since $\mathrm{id}_{M}$ and $\mathrm{id}_{N}$ are harmonic, it follows from Corollary 2.6 that $\mathscr{I}_{\lambda, \varrho}$ is a harmonic map if and only if

$$
\frac{n}{2} d\left(\operatorname{id}_{M}\right)\left[\operatorname{grad}_{M} \lambda^{2}\right]=\mathrm{e}\left(\operatorname{id}_{N}\right)\left(\operatorname{grad}_{M} \varrho^{2}\right) \circ \mathrm{id}_{M}
$$

But

$$
\mathrm{e}\left(\operatorname{id}_{N}\right)=\frac{n}{2}, \quad d\left(\operatorname{id}_{M}\right)\left[\operatorname{grad}_{M} \lambda^{2}\right]=\operatorname{grad}_{M} \lambda^{2}, \quad\left(\operatorname{grad}_{M} \varrho^{2}\right) \circ \mathrm{id}_{M}=\operatorname{grad}_{M} \varrho^{2}
$$

Thus $\Phi_{\lambda, \varrho}$ is harmonic if and only if

$$
\frac{n}{2} \operatorname{grad}_{M} \lambda^{2}-\frac{n}{2} \operatorname{grad}_{M} \varrho^{2}=0
$$

That is,

$$
\operatorname{grad}_{M}\left(\lambda^{2}-\varrho^{2}\right)=0
$$

Since $M$ is assumed to be connected, we get the result.

Example 2.9. (1) Consider $\mathbb{S}^{3} \backslash\{( \pm 1,0,0,0)\}$ with the metric $G$ defined in local coordinates $(t, \theta, \phi)$ by

$$
d s_{G}^{2}=d t^{2}+\sin ^{2} t d \theta^{2}+\sin ^{2} t \sin ^{2} \theta d \phi^{2}
$$

For any real $c<0$, the metric $G^{\prime}$ defined by

$$
d s_{G^{\prime}}^{2}=d t^{2}+\left(\sin ^{2} t-c\right) d \theta^{2}+\left(\sin ^{2} t-c\right) \sin ^{2} \theta d \phi^{2}
$$

is harmonic with respect to $G$. 
Indeed, $\mathbb{S}^{3} \backslash\{(+1,0,0,0)\}$ can be viewed as the warped product of the open interval ] $0, \pi\left[\right.$ and of $\mathbb{S}^{2}$ with warping function $\lambda$ defined on $] 0, \pi[$ by $\lambda(t)=\sin t$.

(2) On the space $\mathbb{H}^{3} \backslash\{(0)\}$ with the metric $G$ defined in local coordinates $(t, \theta, \phi)$ by

$$
d s_{G}^{2}=d t^{2}+\sinh ^{2} t d \theta^{2}+\sinh ^{2} t \sin ^{2} \theta d \phi^{2},
$$

the metric $G^{\prime}$ defined by

$$
d s_{G^{\prime}}^{2}=d t^{2}+\left(\sinh ^{2} t-c\right) d \theta^{2}+\left(\sinh ^{2} t-c\right) \sin ^{2} \theta d \phi^{2} \quad \text { with } c<0
$$

is harmonic with respect to $G$.

Indeed, $\mathbb{U}^{3} \backslash\{(0)\}$ can be viewed as the warped product of the open interval $] 0, \infty[$ and of $\mathbb{S}^{2}$ with warping function $\lambda$ defined on $] 0, \infty[$ by $\lambda(t)=\sinh t$.

2.4. Nonharmonic biharmonic metrics on product spaces. In this paragraph, we study the biharmonicity of the warped product of two identity maps with warping functions of which one is constant.

We obtain then necessary and sufficient conditions for warped metrics to be nonharmonic biharmonic on product spaces.

Before giving the definition of biharmonic metrics, let us point out that, due to the fact that we are interested in nonharmonic biharmonic metrics, we conserve the same places of the domain and the codomain of the identity maps as in the definition of harmonic metrics (see [6]), contrary to the authors in [1] where the places of the domain and codomain are reversed.

Defintion 2.10. Let $(M, g)$ be a Riemannian manifold. A metric $G$ on $M$ is said to be biharmonic with respect to $g$ if the identity id $(M, g) \rightarrow(M, G)$ is a biharmonic map. Let $(M, g)$ and $(N, h)$ be two Riemannian manifolds of dimensions $m$ and $n$, respectively, and $(M \times N, G)$ the Riemannian product of $(M, g)$ and $(N, h)$, respectively; that is, $(M \times$ $N, G)=(M, g) \times(N, h)$.

Let $\varrho \in C^{\infty}(M)$ be a strictly positive function on $M$ and let $G_{\varrho}=\pi^{*} g+(\varrho \circ \pi)^{2} \sigma^{*} h$ be the warped product metric of $g$ and $h$ with warping function $\varrho$.

We have the following result.

THEOREM 2.11. The warped product metric $G_{\varrho}$ is nonharmonic biharmonic with respect to $G$ if and only if

$$
\operatorname{grad} \varrho \neq 0, \quad \Delta_{g} \omega+\frac{n}{4} d\left(\|\omega\|^{2}\right)-2\left(\operatorname{Ric}_{g}\left(\omega^{\sharp}\right)\right)^{b}=0,
$$

with $\omega=d \varrho^{2}$ and $\left(\operatorname{Ric}_{g}\left(\omega^{\sharp}\right)\right)^{b}(X)=\operatorname{Ric}_{g}\left(\omega^{\sharp}, X\right)$, for all $X \in T M$, where $\operatorname{grad} \Delta_{g}=d d^{*}+$ $d^{*} d$, and $\mathrm{Ric}_{g}$ are, respectively, the gradient, the Laplacian, and the Ricci tensor on $(M, g)$.

Proof. By Theorem 2.8 applied to $\lambda \equiv 1$ and the function $\varrho$, the metric $G_{\varrho}$ is nonharmonic with respect to $G$ if and only if $\operatorname{grad} \varrho^{2}=2 \varrho \operatorname{grad} \varrho \neq 0$.

Since $\varrho$ is a strictly positive function, $G_{\varrho}$ is then nonharmonic with respect to $G$ if and only if (i) $\operatorname{grad} \varrho \neq 0$. 
Let $\tau$ be the tension field of the identity map id : $(M \times N, G) \rightarrow\left(M \times N, G_{\varrho}\right)$, and let $J$ be the Jacobi field along id. We have

$$
\tau=-\frac{n}{2} \operatorname{grad} \varrho^{2}, \quad J(\tau)=\Delta^{\mathrm{id}} \tau+\operatorname{trace}_{G} R_{\varrho}(\cdot, \tau),
$$

with $\Delta^{\text {id }} \tau=-\operatorname{trace}_{G}\left(\nabla \nabla \tau-\nabla_{\nabla} \tau\right)$ is the Laplacian on the sections of $T(M \times N)$ and where $R_{\varrho}$ is the Riemannian curvature operator on $\left(M \times N, G_{\varrho}\right)$.

Let $\left(E_{1}, \ldots, E_{m+n}\right)$ be a geodesic orthonormal basis on $T(M \times N)$,

$$
\Delta^{\mathrm{id}} \tau=-\sum_{\hat{I}=1}^{m+n}\left(\nabla_{E_{\hat{I}}} \nabla_{E_{\hat{I}}} \tau-\nabla_{\nabla_{E_{\hat{I}}} E_{\hat{I}}} \tau\right)=-\left(\sum_{\hat{I}=1}^{m} \nabla_{E_{\hat{I}}} \nabla_{E_{\hat{I}}} \tau+\sum_{\hat{I}=m+1}^{m+n} \nabla_{E_{\hat{I}}} \nabla_{E_{\hat{I}}} \tau\right) .
$$

By Lemma 2.1 we have

$$
\sum_{\hat{I}=1}^{m} \nabla_{E_{\hat{I}}} \nabla_{E_{\hat{I}}} \tau=\sum_{\hat{I}=1}^{m} \nabla_{E_{\hat{I}}}^{g} \nabla_{E_{\hat{I}}}^{g} \tau, \quad \text { where } \nabla^{g} \text { is the Levi-Civita connection on }(M, g) \text {. }
$$

Otherwise for $\widehat{I} \in\{m+1, \ldots, m+n\}$,

$$
\nabla_{E_{\hat{I}}} \tau=\frac{\tau \cdot \varrho}{\varrho} E_{\hat{I}} \quad(\text { see }[10]),
$$

and then

$$
\begin{aligned}
\nabla_{E_{\hat{I}}}\left(\nabla_{E_{\hat{I}}} \tau\right)= & \nabla_{E_{\hat{I}}}\left(\frac{\tau \cdot \varrho}{\varrho} E_{\hat{I}}\right) \\
= & \left(\frac{\tau \cdot \varrho}{\varrho}\right) \nabla_{E_{\hat{I}}} E_{\hat{I}}+\left(E_{\hat{I}} \cdot \frac{\tau \cdot \varrho}{\varrho}\right) E_{\hat{I}} \\
= & \frac{\tau \cdot \varrho}{\varrho}\left[-\varrho\left\langle E_{\hat{I}}, E_{\hat{I}}\right\rangle \operatorname{grad} \varrho+\nabla_{E_{\hat{I}}}^{h} E_{\hat{I}}\right], \\
& \text { since } E_{\hat{I}} \cdot \frac{\tau \cdot \varrho}{\varrho}=0 \text { and by Lemma } 2.1 \\
= & n\|\operatorname{grad} \varrho\|^{2} \varrho h_{\hat{I}} \text { grad } \varrho, \quad \text { since } \nabla_{E_{\hat{I}}}^{h} E_{\hat{I}}=\sigma_{*}\left(\nabla_{E_{\hat{I}}} E_{\hat{I}}\right)=0, \\
& \text { where } \nabla^{h} \text { is the Levi-Civita connection on }(N, h) .
\end{aligned}
$$

Thus

$$
\sum_{\hat{I}=m+1}^{m+n} \nabla_{E_{\hat{I}}} \nabla_{E_{\hat{I}}} \tau=\frac{n^{2}}{2}\|\operatorname{grad} \varrho\|^{2} \operatorname{grad} \varrho^{2} .
$$

We obtain then

$$
\Delta^{\mathrm{id}} \tau=-\sum_{\hat{I}=1}^{m} \nabla_{E_{\hat{I}}}^{g} \nabla_{E_{\hat{I}}}^{g} \tau-\frac{n^{2}}{2}\|\operatorname{grad} \varrho\|^{2} \operatorname{grad} \varrho^{2} .
$$


On the other hand,

$$
\begin{aligned}
\sum_{\hat{I}=1}^{m+n} R_{\varrho}\left(E_{\hat{I}}, \tau\right) E_{\hat{I}}= & \sum_{\hat{I}=1}^{m} R_{\varrho}\left(E_{\hat{I}}, \tau\right) E_{\hat{I}}+\sum_{\hat{I}=m+1}^{m+n} R_{\varrho}\left(E_{\hat{I}}, \tau\right) E_{\hat{I}} \\
= & \sum_{\hat{I}=1}^{m} R_{g}\left(E_{\hat{I}}, \tau\right) E_{\hat{I}}+\sum_{\hat{I}=m+1}^{m+n} \varrho<E_{\hat{I}}, E_{\hat{I}}>\nabla_{\tau}(\operatorname{grad} \varrho), \\
& \text { by }[10, \text { Proposition } 40, \text { page } 210] \\
= & \sum_{\hat{I}=1}^{m} R_{g}\left(E_{\hat{I}}, \tau\right) E_{\hat{I}}+n \varrho \nabla_{\tau}(\operatorname{grad} \varrho) \\
= & -\sum_{\hat{I}=1}^{m} R_{g}\left(\tau, E_{\hat{I}}\right) E_{\hat{I}}-\frac{n^{2} \varrho^{2}}{2} \operatorname{grad}\left(\|\operatorname{grad} \varrho\|^{2}\right),
\end{aligned}
$$

since

$$
\begin{gathered}
\nabla_{\tau}(\operatorname{grad} \varrho)=-\frac{n}{2} \nabla_{\operatorname{grad} \varrho^{2}}(\operatorname{grad} \varrho)=-n \varrho \nabla_{\operatorname{grad} \varrho}(\operatorname{grad} \varrho), \\
\nabla_{\operatorname{grad} \varrho}(\operatorname{grad} \varrho)=\frac{1}{2} \operatorname{grad}\left(\|\operatorname{grad} \varrho\|^{2}\right) .
\end{gathered}
$$

It follows

$$
\begin{aligned}
J(\tau)=-\frac{n}{2}\{ & -\sum_{\hat{I}=1}^{m} \nabla_{E_{\hat{I}}}^{g} \nabla_{E_{\hat{I}}}^{g} \operatorname{grad} \varrho^{2}-\sum_{\hat{I}=1}^{m} R_{g}\left(\operatorname{grad} \varrho^{2}, E_{\hat{I}}\right) E_{\hat{I}} \\
& \left.+n\|\operatorname{grad} \varrho\|^{2} \operatorname{grad} \varrho^{2}+n \varrho^{2} \operatorname{grad}\left(\|\operatorname{grad} \varrho\|^{2}\right)\right\} \\
=- & \frac{n}{2}\left\{-\sum_{\hat{I}=1}^{m} \nabla_{E_{\hat{I}}}^{g} \nabla_{E_{\hat{I}}}^{g} \operatorname{grad} \varrho^{2}-\sum_{\hat{I}=1}^{m} R_{g}\left(\operatorname{grad} \varrho^{2}, E_{\hat{I}}\right) E_{\hat{I}}\right. \\
& \left.+\frac{n}{4} \operatorname{grad}\left(\left\|\operatorname{grad} \varrho^{2}\right\|^{2}\right)\right\},
\end{aligned}
$$

since

$$
\|\operatorname{grad} \varrho\|^{2} \operatorname{grad} \varrho^{2}+\varrho^{2} \operatorname{grad}\left(\|\operatorname{grad} \varrho\|^{2}\right)=\frac{1}{4} \operatorname{grad}\left(\left\|\operatorname{grad} \varrho^{2}\right\|^{2}\right) .
$$

Thus $G_{\varrho}$ is biharmonic with respect to $G$ if and only if

$$
-\sum_{\widehat{I}=1}^{m} \nabla_{E_{\widehat{I}}}^{g} \nabla_{E_{\widehat{I}}}^{g} \operatorname{grad} \varrho^{2}-\sum_{\widehat{I}=1}^{m} R_{g}\left(\operatorname{grad} \varrho^{2}, E_{\widehat{I}}\right) E_{\widehat{I}}+\frac{n}{4} \operatorname{grad}\left(\left\|\operatorname{grad} \varrho^{2}\right\|^{2}\right)=0 .
$$


By the Weitzenböck formula,

$$
\Delta_{g} \omega=-\operatorname{trace} \nabla_{g}^{2} \omega+\left(\operatorname{Ric}_{g}\left(\omega^{\sharp}\right)\right)^{b},
$$

where $\#$ and $b$ are the usual musical isomorphisms.

Hence the relation $\left(^{*}\right)$ is equivalent to

(ii)

$$
\Delta_{g} \omega+\frac{n}{4} d\left(\|\omega\|^{2}\right)-2\left(\operatorname{Ric}_{g}\left(\omega^{\sharp}\right)\right)^{b}=0 .
$$

From (i) and (ii) we get the result.

When $(M, g)$ is Einstein, the one-form $\left(\operatorname{Ric}_{g}\left(\omega^{\sharp}\right)\right)^{b}$ is proportional to $\omega$. Thus as an immediate consequence of the previous theorem, we have the following.

Corollary 2.12. Let $\varrho \in C^{\infty}(M), \varrho>0$, with $\operatorname{grad} \varrho \neq 0$. Assume that $(M, g)$ is an Einstein manifold and that $\operatorname{grad}(\|\operatorname{grad} \varrho\|)$ is parallel to $\operatorname{grad} \varrho$. If $G_{\varrho}$ is harmonic with respect to $G$, then $\varrho$ is an isoparametric function on $M$.

Recall that a function $f$ on $M$ is said to be isoparametric if there exist real functions $\alpha$ and $\beta$ such that $\|d f\|^{2}=\alpha \circ f$ and $\Delta f=\beta \circ f$ or equivalently if $\operatorname{grad}(\|\operatorname{grad} f\|)$ and $\operatorname{grad}(\Delta f)$ are parallel to grad $f$, with grad $f \neq 0$ (see $[1,2]$ for more information about isoparametric functions).

\section{Acknowledgments}

The author thanks warmly the Abdus Salam International Centre for Theoretical Physics for its support during this work. Thanks are due to the referee for his helpful remarks and suggestions leading to consistent improvement of this paper.

\section{References}

[1] P. Baird and D. Kamissoko, On constructing biharmonic maps and metrics, Annals of Global Analysis and Geometry 23 (2003), no. 1, 65-75.

[2] A. Balmuş, Biharmonic properties and conformal changes, Analele Ştiinţifice ale Universitătii "Al. I. Cuza" din Iaşi. Serie Nouă. Matematică 50 (2004), no. 2, 361-372 (2005).

[3] R. Caddeo, S. Montaldo, and C. Oniciuc, Biharmonic submanifolds of $\mathbb{S}^{3}$, International Journal of Mathematics 12 (2001), no. 8, 867-876.

[4] _ Biharmonic submanifolds in spheres, Israel Journal of Mathematics 130 (2002), 109123.

[5] R. Caddeo, S. Montaldo, and P. Piu, On Biharmonic Maps, Global Differential Geometry: The Mathematical Legacy of Alfred Gray (Bilbao, 2000), Contemporary Mathematics, vol. 288, American Mathematical Society, Rhode Island, 2001, pp. 286-290.

[6] B.-Y. Chen and T. Nagano, Harmonic metrics, harmonic tensors, and Gauss maps, Journal of the Mathematical Society of Japan 36 (1984), no. 2, 295-313.

[7] J. Eells Jr. and J. H. Sampson, Harmonic mappings of Riemannian manifolds, American Journal of Mathematics 86 (1964), no. 1, 109-160.

[8] G. Y. Jiang, 2-harmonic isometric immersions between Riemannian manifolds, Chinese Annals of Mathematics. Series A 7 (1986), no. 2, 130-144.

[9] - 2-harmonic maps and their first and second variational formulas, Chinese Annals of Mathematics. Series A 7 (1986), no. 4, 389-402. 
[10] B. O'Neill, Semi-Riemannian Geometry, Pure and Applied Mathematics, vol. 103, Academic Press, New York, 1983.

[11] C. Oniciuc, Biharmonic maps between Riemannian manifolds, Analele Ştiinţifice ale Universitătii “Al. I. Cuza" din Iaşi. Serie Nouă. Matematică 48 (2002), no. 2, 237-248 (2003).

[12] Z. Sentürk, On warped product manifolds, Proceedings of International Conference on Differential Geometry and Its Applications, Prague, 2005, pp. 109-117.

Leonard Todjihounde: Institut de Mathematiques et de Sciences Physiques, Université Nationale du Benin, BP 613, EP 2628 Porto Novo, Benin

E-mail address: leonardt@imsp-uac.org 


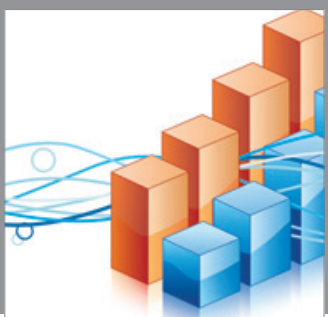

Advances in

Operations Research

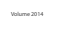

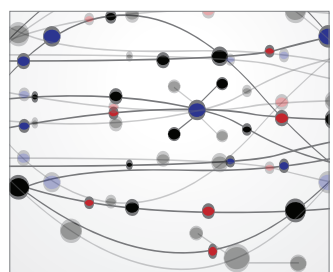

\section{The Scientific} World Journal
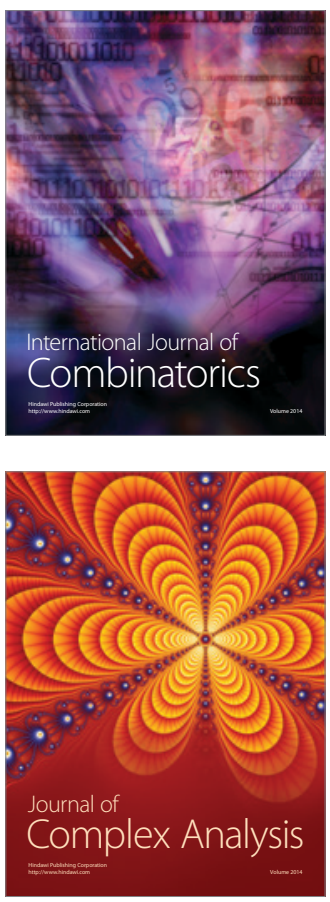

International Journal of

Mathematics and

Mathematical

Sciences
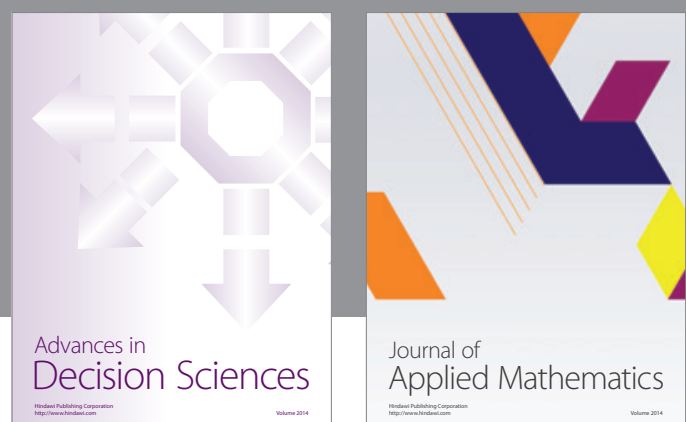

Journal of

Applied Mathematics
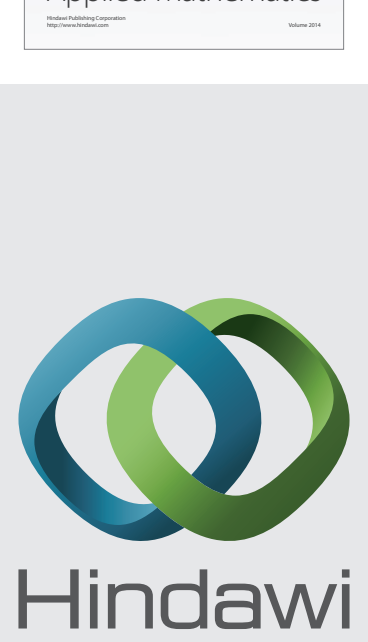

Submit your manuscripts at http://www.hindawi.com
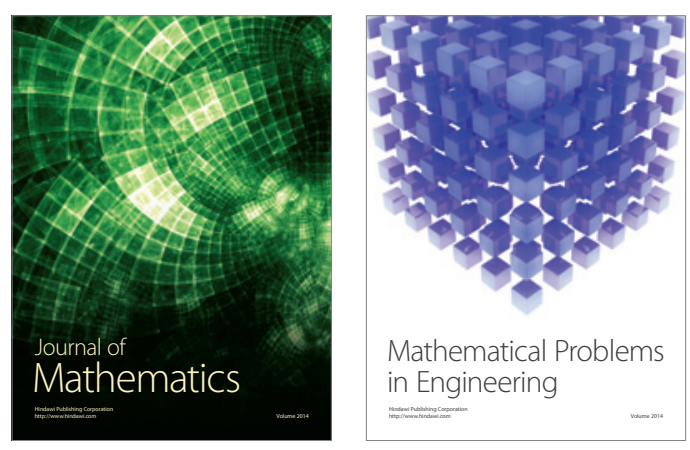

Mathematical Problems in Engineering
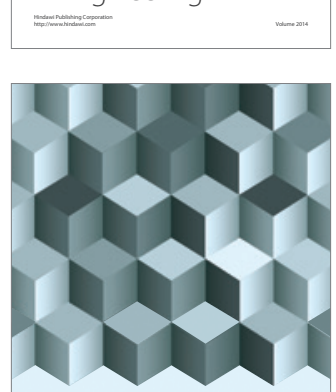

Journal of

Function Spaces
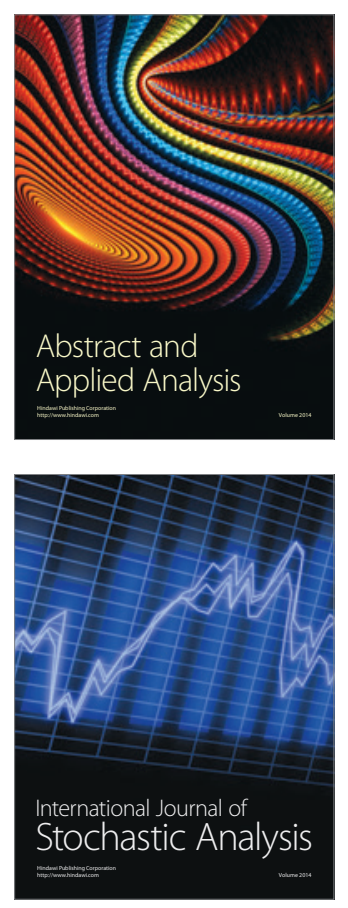

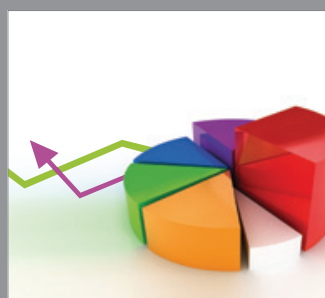

ournal of

Probability and Statistics

Promensencen
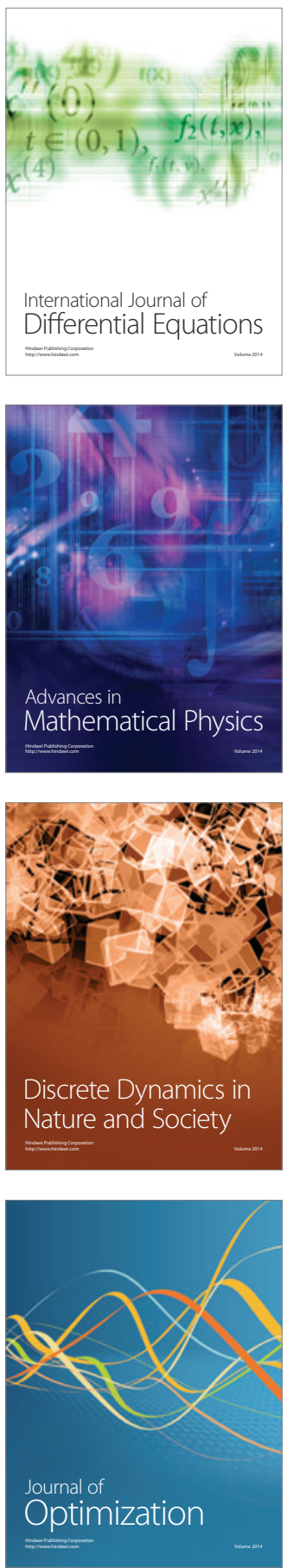